\title{
Tumor Lysis Syndrome in the Course of Burkitt Lymphoma Revealed by Medullar Compression
}

\author{
L. Boukassa1 ${ }^{*}$, S. B. Kinata Bambino', O. B. Ngackosso', K. Mavoungou Biatsi'1, \\ H. B. Ekouele-Mbaki'1, P. Mawanza²
}

\begin{abstract}
${ }^{1}$ Department of Surgery, Division of Neurosurgery, Brazzaville Academic Hospital, Brazzaville, Republic of Congo ${ }^{2}$ Department of Anesthesia-Reanimation, Brazzaville Academic Hospital, Brazzaville, Republic of Congo

Email: ^lboukassalouf@gmail.com
\end{abstract}

How to cite this paper: Boukassa, L., Bambino, S.B.K., Ngackosso, O.B., Biatsi, K.M., Ekouele-Mbaki, H.B. and Mawanza, P. (2019) Tumor Lysis Syndrome in the Course of Burkitt Lymphoma Revealed by Medullar Compression. Open Journal of Modern Neurosurgery, 9, 429-435. https://doi.org/10.4236/ojmn.2019.94040

Received: July 31, 2019

Accepted: October 8, 2019

Published: October 11, 2019

Copyright $\odot 2019$ by author(s) and Scientific Research Publishing Inc. This work is licensed under the Creative Commons Attribution International License (CC BY 4.0).

http://creativecommons.org/licenses/by/4.0/

\begin{abstract}
Background: Tumor lysis syndrome is unknown to many neurosurgical teams. A dreaded complication, especially in the course of hematological malignancies, like it was the case in two of our patients operated for spinal cord compression by Burkitt's lymphoma. Objectives: To describe the clinical and biological presentation of this potentially fatal complication, which can be improved by early preventive or curative treatments. Case Reports: Patient 1: A 49-year-old woman was admitted for vertebral and intercostal pain and paraparesis. Myelography revealed epidural medullary compression at the 7 th thoracic vertebrae (Th7) level. Patient 2: A 67-year-old woman was admitted for cervico-thoracic pain and paraparesis. MRI showed posterior epidural medullary compression between the 1st thoracic vertebrae (Th1) and 5th thoracic vertebrae (Th5). In both cases, an urgent decompressive laminectomy was performed. They had also received postoperative corticosteroid therapy. The multi-visceral failure occurred on the 1st day (D1) and 3rd day (D3) postoperatively, respectively. In the absence of adequate management, the outcome was fatal for the two patients respectively at D3 and D5 postoperatively. This syndrome was retained in the presence of glucocorticoid (triggering factor) and the histological result in favor of Burkitt lymphoma (target disease). Conclusion: Malignant hemopathies is a common cause of spinal cord compressions. From these two cases, the importance of biological and clinical surveillance emerges, with patients requiring urgent management for radiculo-medullar tumor compression.
\end{abstract}

\section{Keywords}

Tumor Lysis Syndrome, Spinal Cord Compression, Burkitt Lymphoma 


\section{Introduction}

Tumor lysis syndrome (TLS) includes all clinical and/or biological disturbances, secondary to the brutal and massive lysis of tumor cells of certain malignancies characterized by a rapid resolution time and a strong tumor mass [1] [2]. Burkitt lymphoma is a rare cause of spinal cord compression, especially as a mode of revelation. It is part, with other hematological malignancies and some solid tumors, of conditions that may present this complication. This syndrome is often triggered after starting the treatment of these conditions (chemotherapy, radiotherapy, corticosteroids and other treatments) [3] [4]. Spontaneous cases have also been described. Well known in oncology, this syndrome is ignored by many neurosurgical teams and yet it complicates both solid malignant tumors and malignant hemopathies, involving the latter. If in patients with conditions that cause this syndrome, its prevention is started early with favorable developments. In other cases, the unfavorable outcome of this serious syndrome, made this diagnosis during the mortality audit. This was the case of the two patients whose observations we report.

The aim of these reports is to describe the presentation, treatment and outcome of these patients and to highlight this potentially fatal condition which can be improved by early preventive or curative treatments.

\section{Case Reports}

\subsection{Case 1}

Mrs. K. D., aged 49, was admitted on June 23, 2007 to Brazzaville University Hospital for average thoracic spinal pain. She had no history of infectious or tumoral pathology. She did not smoke, drink alcohol and, had no known allergy. These pains radiated to the lower thoracic belt, were associated with heaviness of the lower limbs, causing intermittent neurological claudication, and gradual reduction of the perimeter. These symptoms had been worsening for a week. There was no notion of fever or alteration of the general condition.

Clinical examination of this patient indicated hypoesthesia of higher Th6 level, paraparesis rated at $3 / 5$, vivacity of osteo-tendinous reflexes, bilateral Babinski sign and urine retention. The conclusion of this review was Th6 bone marrow compression syndrome. Myelography revealed an epidural medullary compression in Th7. She underwent urgent surgical management (decompressive laminectomy from Th7 to Th10 associated with the removal of the compressive tumor epiduritis). This epidural material was made of greyish, friable and hemorrhagic tissue, maximum in Th8 and Th9, also invading neural arches and paravertebral muscles. She had received, immediately postoperatively, $360 \mathrm{mg}$ of methyl prednisolone. While the clinical course was neurologically stable, the patient had a rapidly progressive onset of day 1 postoperative, with polyvisceral failure in a febrile environment at $39.2^{\circ} \mathrm{C}$ and altered consciousness. The biological assessment showed hyperkalemia at $7.7 \mathrm{mmol} / \mathrm{l}$, hypocalcemia at $5.12 \mathrm{mmol} / \mathrm{l}$, hyperuria at $543 \mu \mathrm{mol} / \mathrm{l}$, hypephosphatemia at $5.57 \mathrm{mmol} / \mathrm{l}$. Creatinine was 131 
$\mathrm{mg} / \mathrm{l}$ and urea was $17.2 \mathrm{mmol} / \mathrm{l}$. The evolution was lethal at day 3 postoperative.

The anatomopathological result was in favor of Burkitt-type non-Hodgkin's lymphoma.

\subsection{Case 2}

Mrs. H.C., aged 67, was admitted to the University Hospital of Brazzaville on 23 September 2013 for cervico-thoracic pain and motor deficit of the lower limbs. These signs had been evolving for three weeks. No history of infectious or tumoral pathology was reported. There was no notion of taking alcohol or tobacco and had no known allergy. The physical examination of this female patient had noted paraparesis at 2/5 proximal and 3/5 distal, hypoesthesia Th4 level, bladder globe, vivacity of osteo-tendinous reflexesand Babinski sign on the right. It was therefore a Th4 bone marrow compression syndrome. Spino-medullary MRI showed posterior epidural medullary compression between Th1 and Th5.

She had been operated on urgently. A decompressive laminectomy of Th1 to Th5 was performed. The compressive tissue was in the posterior epidural space, especially in Th3. It was purplish gray, friable, and slightly haemorrhagic. Bone tissue and muscles were spared. The decompression was considered satisfactory and the material preserved for histological examination. She received immediate postoperative $240 \mathrm{mg}$ of methyl prednisolone intravenous. The evolution was marked by the appearance of a fever at $38.7^{\circ} \mathrm{C}$ on the second day post-operative. Para-clinical assessment linked this picture to pneumonia associated with urinary $E$. coli infection and the patient being treated with antibiotics. Clinical degradation continued despite antibiotic therapy. On Day 3 post-operative, the patient had multiple organs involvement with dyspnea, oligo-anuria, major disturbances of heart rate and blood pressure, and impaired consciousness in a febrile context $\left(40.5^{\circ} \mathrm{C}\right)$. The laboratory results showed hyperkalemia $(8.7 \mathrm{mmol} / \mathrm{l})$, hypocalcaemia $(3.12 \mathrm{mmol} / \mathrm{l})$, hyperuricemia $(642 \mu \mathrm{mol} / \mathrm{l})$ and hyperphosphatemia $(5.49 \mathrm{mmol} / \mathrm{l})$, creatinine was $131 \mathrm{umol} / \mathrm{l}$ and urea $16.3 \mathrm{mmol} / \mathrm{l}$. Despite the resuscitative measures undertaken, the outcome was quickly fatal. The death occurred on Day 5 post-operative in a febrile multiple organs failure. The histological result was in favor of Burkitt-type non-Hodgkin's lymphoma.

\section{Discussion}

Spinal cord compressions are neurosurgical emergencies that have benefited from the contribution of new medical imaging techniques in general and MRI particularly. Meanwhile, histology remains essential in the etiological diagnosis of the latter. Medullary compression, as a mode of revelation of Burkitt's lymphoma, is a rare event. Sess [5] relates two and Gnonlonfoun [6] one case. Usually characterized by an important tumoral syndrome of abdominal and/or craniofacial localization, it reaches the vertebro-radiculo-medullary sector in the advanced stages [6]. In all these cases, these are epidural localizations. The surgery is aimed at rapid radiculo-medullary decompression to preserve the functional prognosis. It allows to find an epidural compressive material often greyish 
friable and haemorrhagic just evoking a tumor epiduritis. Even when spinal compression is the mode of revelation, the patient remains exposed to TLS.

This complication, suspected since the 18th century during leukaemias; is currently very well known to oncologists, because encountered during several malignant diseases especially hematological [1]. It groups together the set of hydroelectrolytic and metabolic disorders observed mainly, but not exclusively, during malignant diseases with fast and high-mass splitting times. These elements characterize Burkitt's lymphoma which is an LMNH. Cairo and Bishop [3] classify these TLS in biological and clinical. In biological TLS, patients only carry biological disturbances such as hyperuricemia, hyperkalemia, hyperphosphoremia and hypocalcemia. These disturbances should be at least $25 \%$ of normal value and should be observed within 3 days before and 7 days after initiation of treatment. The clinical forms associated with these biological forms, at least three major clinical manifestations related to the toxicity of these substances namely: renal failure, cardiac arrhythmia, comitiale crisis and sudden death [3] [4] [7]. It should be noted, as Wilson [8] points out, that this classification does not take into account syndromes of spontaneous tumor lysis.

Our two cases were clinical forms since biological disturbances were associated with clinical manifestations. The age of onset of this syndrome and sexual prevalence are not found in the different studies. Both cases involved women aged 67 and 49 years. The majority of studies report an incidence ranging between $4.4 \%$ to $42 \%$ for biological TLS and $5 \%$ to $9.8 \%$ for clinical forms [3] [4]. This incidence is important in haematological malignancies [7] [9]. In a study that included 788 patients with LMNH and acute leukemia, the incidence of hyperuricemia and TLS was $18.9 \%$ and $5 \%$, respectively [1]. Mortality in leukemia and in LMNH was estimated at $1.9 \%$.

Cases of SLT have also been reported during some solid malignant tumors such as hepatoblastomas, hepatocellular carcinomas, breast adenocarcinomas and vulvar carcinomas [2] [10]. Solid neurosurgical tumors can also be complicated by TLS. These are sacro-coccygeal teratomas, neuroblastomas and medulloblastomas. But this complication seems less common in solid tumors, Gemici [2] lists only 45 cases over a period of 26 years. The factors that trigger this TLS are multiple. Although cancer, chemotherapy and radiotherapy are the most incriminated, embolization, surgery and fever are cited. Corticosteroid therapy even at low doses can cause it [11] [12] [13]. Spontaneous evolutions have also been reported [9] [14] [15] [16]. The mechanisms of occurrence in these spontaneous cases are not yet well understood. In our patients, two factors are found: surgery and corticotherapy. Indeed these two patients with spinal cord compressions, neurosurgical emergencies, had been operated in this context. The corticosteroid therapy that we routinely administer to our patients in these cases aims to fight against inflammatory phenomena.

Indeed, the lysis of tumor malignant cells, induced by these treatments or spontaneously, causes the release in the extracellular medium of the cellular contents. These substances in large quantities are: electrolytes (potassium and phos- 
phorus) and metabolites of certain substances (uric acid for nucleic acids). It will follow a passing of renal excretory capacities and reuse of the organism. We will observe various disturbances whose cardinal are: hyperkalemia, hyperphosphoremia, hypocalcemia and hyperuricemia. The consequences of these disturbances are renal, cardiac, muscular and encephalic [4] [9] [11] [14] [16]. The symptomatology is that of an attack, rapidly progressive, renal, cardiac, muscular and encephalic, in a patient carrying a target pathology, identified or not [7].

The clinical manifestations consist of nausea and vomiting, tetany, seizures, asthenia, oligoanuria, cardiac arrhythmias, disorders of the higher functions. The electrical abnormalities observed are tachyarrhythmia, the lengthening of the interval QT and the modification of the wave T. These tables are associated randomly and are fast. A biological assessment for hydroelectrolytic and metabolic disorders must be performed. Early therapeutic management should be implemented and, if not known, the search for the target pathology undertaken.

The treatment is preventive and curative [2] [7] [8] [11]. Preventive treatment is for patients with the target pathologies. The purpose of this treatment is to avoid the appearance of hydroelectrolytic and metabolic disturbances spontaneously or after the start of an anti-cancer treatment. It is based on hyper hydration, alkalinization of urine, the use of hypouricémiants. The curative treatment will combine, in addition to these means mentioned above, the management of hydroelectrolytic and metabolic disorders. In cases of severe renal failure, the use of dialysis becomes essential.

\section{Conclusion}

Burkitt's lymphoma remains a rare cause of spinal cord compression. Whatever its mode of revelation is, the TLS is one of the evolutionary possibilities. The severity of the latter justifies the start of diagnostic means, especially in patients with target pathologies such as haematological malignancies. Early treatment in a multidisciplinary setting, about consequence of this early diagnosis, improves the prognosis of this complication.

\section{Declaration}

None.

\section{Author's Contributions}

L Boukassa wrote the article

\section{Funding}

None.

\section{Acknowledgements}

A special thank you to Dr JC Moundzika-Kibamba for his contribution to this article. 


\section{Conflicts of Interest}

The authors declare no conflicts of interest regarding the publication of this paper.

\section{References}

[1] Gemici, C. (2006) Tumor Lysis Syndrome in Solid Tumors. Clinical Oncology, 18, 773-780. https://doi.org/10.1016/j.clon.2006.09.005

[2] Jabbour, E. and Ribrag, V. (2005) Current Treatment of Tumor Lysis Syndrome. The Journal of Internal Medicine, 26, 27-32. https://doi.org/10.1016/j.revmed.2004.06.014

[3] Cairo, M.S. and Bishop, M. (2004) Tumor Lysis Syndrome: New Therapeutic Strategies and Classification. British Journal of Haematology, 127, 3-11. https://doi.org/10.1111/j.1365-2141.2004.05094.x

[4] Hande, K.R. and Garrow, G.C. (1993) Acute Tumor Lysis Syndrome in Patients with High-Grade Non-Hodgkin's Lymphoma. The American Journal of Medicine, 94, 133-139. https://doi.org/10.1016/0002-9343(93)90174-N

[5] Sess, E., N'dri-Oka, D., Varlet, G., Koffi, K., Boni, N.R. and Ba Zeze, V. (2001) Medullary Compression by Burkitt's Lymphoma: Analysis of 7 Observations. Neurosurgery, 47, 552-556.

[6] Gnonlonfoun, D., Adjien, K.C., Yekpe, P., Ossou-Nguiet, P.M., Adoukonou, T.A., Ndiaye, M., et al. (2011) Slow Medullary Compression Secondary to Intracerebral Burkitt Lymphoma. African Journal of Neurological Sciences, 30, 76-81.

[7] Raul, C.R. and Ching-Hon, P. (2003) Recombinant Urate Oxidase for Prevention of Hyperuricemia and Tumor Lysis Syndrome in Lymphoid Malignancies. Clinica Lymphoma, 3, 225-232. https://doi.org/10.3816/CLM.2003.n.003

[8] Wilson, F.P. and Berns, J.S. (2014) Tumor Lysis Syndrome: New Challenges and Recent Advances. Advances in Chronic Kidney Diseases, 21, 18-26.

https://doi.org/10.1053/j.ackd.2013.07.001

[9] Firwana, B.M., Rim Hasan, R., Hasan, N., Alahdab, F., Alnahhas, I., Hasan, S., et al. (2012) Tumor Lysis Syndrome: A Systematic Review of Case Series and Case Reports. Postgraduate Medicine, 124, 92-101. https://doi.org/10.3810/pgm.2012.03.2540

[10] Mirrakhimov, A.E., Ali, A.M., Khan, M. and Barbaryan, A. (2014) Tumor Lysis Syndrome in Solid Tumor: An Up to Date Review of the Literature. Rare Tumors, 6, 5389. https://doi.org/10.4081/rt.2014.5389

[11] Cairo, M.S., Hairdresser, B., Reiter, A. and Younes, A. (2010) Recommendations for the Evaluation of Risk and Prophylaxis of Tumor Lysis Syndrome (TLS) in Adults and Children with Malignant Diseases: An Expert TLS Panel Consensus. British Journal of Haematology, 149, 578-586. https://doi.org/10.1111/j.1365-2141.2010.08143.x

[12] Kim, J.O., Jun, D.W., Tae, H.J., Lee, K.N., Lee, O.Y., Choi, H.S., et al. (2015) Low-Dose Steroid-Induced Tumor Lysis Syndrome in a Hepatocellular Carcinoma Patient. Clinical and Molecular Hepatology, 21, 85-88. https://doi.org/10.3350/cmh.2015.21.1.85

[13] Kopterides, P., Lignos, M., Mavrou, I. and Armaganidis, A. (2005) Steroid Induced Tumor Lysis Syndrome in a Patient with Mycosis Fungoides Treated for Presumed Pneumocystis carinii Pneumonia. American Journal of Hematology, 80, 309. 
https://doi.org/10.1002/ajh.20444

[14] Chanimov, M., Koren-Michowitz, M., Cohen, M.L., Pilipodi, S. and Bahar, M. (2006) Tumor Lysis Syndrome Induced by Dexamethasone. Anesthesiology, 105, 633-634. https://doi.org/10.1097/00000542-200609000-00042

[15] Ji, Y.L., Sung, H.L., Ji, Y.L., Ji, H.K., Ki, H.C., Keunchil, P., et al. (2013) Tumor Lysis Syndrome in a Solid Tumor. Case Report of a Patient with Invasive Thymoma. Cancer Research and Treatment, 45, 343-348. https://doi.org/10.4143/crt.2013.45.4.343

[16] Mirrakhimov, A.E., Voore, P., Khan, M. and Ali, A.M. (2015) Tumor Lysis Syndrome: A Clinical Review. World Journal of Critical Care Medicine, 4, 130-138. https://doi.org/10.5492/wjccm.v4.i2.130 